Network Working Group

Request for Comments: 4729

Category: Informational
M. Abel

NEC Forum

November 2006

\title{
A Uniform Resource Name (URN) Namespace for \\ the Near Field Communication (NFC) Forum
}

Status of This Memo

This memo provides information for the Internet community. It does not specify an Internet standard of any kind. Distribution of this memo is unlimited.

Copyright Notice

Copyright (C) The IETF Trust (2006).

Abstract

This document describes the Namespace Identifier (NID) for Uniform Resource Name (URN) resources published by the Near Field

Communication (NFC) Forum. The NFC Forum defines and manages resources that utilize this URN identification model. Management activities for these and other resource types are provided by the NFC Forum Technical Committee.

Table of Contents

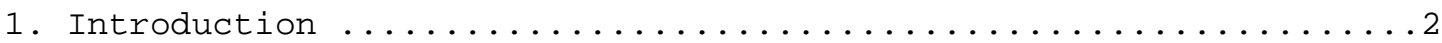

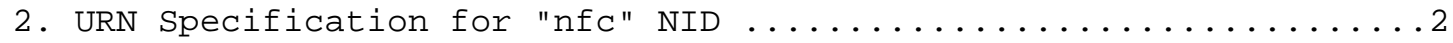

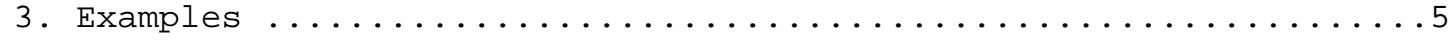

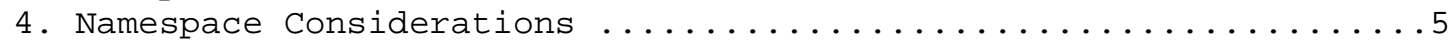

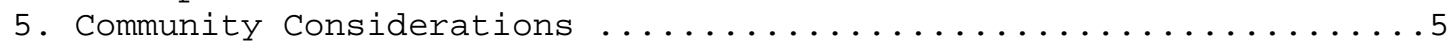

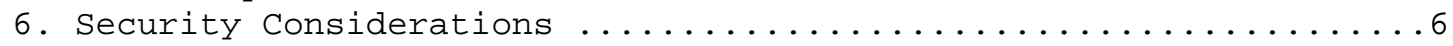

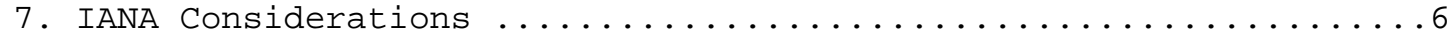

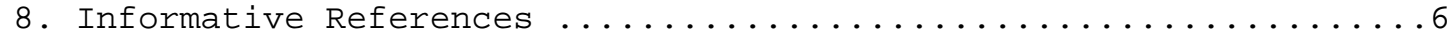

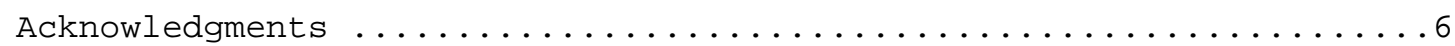




\section{Introduction}

The NFC Forum is a specification development body developing technologies related to near-field communications. This activity is supported by a membership comprised of chip vendors, smart card vendors, equipment vendors, software developers, finance and banking service providers, content providers, and other interested companies.

Some of the technologies being developed by the NFC Forum need namespaces that are managed so that they are unique and persistent. To ensure that the uniqueness is absolute, the registration of a specific NID for use by the NFC Forum was deemed appropriate. Therefore, a full and complete registration will follow the namespace specification process as defined in [RFC3406].

2. URN Specification for "nfC" NID

Namespace ID:

$\mathrm{nfC}$

Registration Information:

Registration version number: 1

Registration date: 2006-05-17

Declared registrant of the namespace:

Registering organization

Name: Near Field Communication Forum, Inc.

Address: 401 Edgewater Place, Suite 600

Wakefield, MA 01880

Designated contact

Role: Technical Program Manager

Email: TPMenfc-forum.org

Declaration of syntactic structure:

The Namespace Specific String (NSS) of all URNs that use the "nfC" NID will have the following structure:

urn:nfc: $\{$ NFCresource $\}:\{$ ResourceSpecificString $\}$

Where the "\{NFCresource $\}$ " is a US-ASCII string that conforms to the URN syntax requirements [RFC2141] and defines a specific class of resource. Each resource class has a specific labeling scheme 
that is covered by "\{ResourceSpecificString\}" which also conforms to the naming requirements of [RFC2141].

NFC Forum working groups will manage the assignment of NFC resources and the specific registration values assigned for each resource class. The Technical Committee will coordinate creation of new resource class assignments based on community need.

Relevant ancillary documentation:

The NFC Forum publishes specifications describing the use of near-field communication for interoperable exchange of information between devices in close proximity. Among these specifications are the designation of new data types, schema, XML elements and attributes, protocols, and other formally named items intended for machine parsing. Interested parties are referred to the NFC Forum web site where these publications will be made available to the community:

http://www.nfc-forum.org/

Identifier uniqueness considerations:

The NFC Forum working groups and Technical Committee will ensure uniqueness of resource names assigned by the NFC Forum within the resource classes for which they are responsible.

When authority and responsibility for assignment of names with a resource class are delegated to an external organization, commitment to adhere to the uniqueness requirements of the assigned resource names will be a pre-condition of such delegation.

The structure of the NFC Forum namespace into resource classes will further ensure isolation of names in each class from names in other classes.

It is anticipated that some resource classes may be open to selfassignment by any interested individual or organization. To ensure a degree of uniqueness for these self-assigned resource names, fully-qualified domain names will be factored into the name to distinguish the naming authorities.

It should be understood that due to the flexibility of the domain name system and the underlying market-based forces that allow for ownership transfer and abandonment of domain names, no guarantee of long-term uniqueness or persistence can be made for this class 
of resource names beyond that made for the domain names themselves.

Identifier persistence considerations:

The NFC Forum will not reassign names and will thus guarantee the persistence of names beyond the expected lifetime of the named resource. NFC Forum does not anticipate operating a name resolution service for the assigned names and does not anticipate any other usability issues of the assigned names beyond those for any other naturally aging technology.

Process of identifier assignment:

The NFC Forum will provide procedures for registration of each class of resource that it maintains. Each such resource may have three types of registration activities:

1) Registered names associated with NFC Forum specifications or services;

2) Registration of names or resource classes for other entities;

3) Name models for use in experimental purposes.

Process for identifier resolution:

The namespace is not listed with a Resolution Discovery system $(\mathrm{RDS})$; this is not relevant.

Rules for Lexical Equivalence:

No special considerations; the rules for lexical equivalence of [RFC2141] apply.

Conformance with URN Syntax:

No special considerations.

Validation mechanism:

None specified. URN assignment and registration will be handled by procedures implemented in support of NFC Forum activities.

Scope:

Global 
3. Examples

The following examples are representative URNs that could be assigned by the NFC Forum. They may not be the actual strings that would be assigned:

urn: nfc:wkt:Uri

Defines the well-known type identifier used to identify URI bookmark data objects defined by the NFC Forum and exchanged by NFC devices.

urn:nfc:schema:manifest

Declares an XML namespace for elements and attributes, used in an XML schema to describe an NFC Forum data transfer manifest.

4. Namespace Considerations

The NFC Forum is developing a variety of communication protocol and data exchange specifications. Some of these specifications depend upon supporting information (e.g., data descriptions, attributes, schema, etc.) to be fully specified. For proper operation, descriptions of the needed supporting information must exist and be available in a unique, reliable, and persistent manner. These dependencies provide the basis of need for namespaces, in one form or another.

As type information is relayed across NFC Forum protocols, the need for compact, machine-parsed type identification of data and metacontent, with the attributes of uniqueness and reliability mentioned above, becomes imperative for proper interoperation across widely varied implementations.

As the NFC Forum work is ongoing and covers many technical areas, the possibility of using various other namespace repositories has been deemed impractical. Each data object, description, or schema as defined in the NFC Forum could possibly be related to multiple different other namespaces, so further conflicts of association might occur. Thus, the intent is to utilize the requested URN namespace to manage NFC Forum-defined objects, descriptions, and schema.

\section{Community Considerations}

The objects and descriptions required for specifications produced and published by the NFC Forum are generally available for use by other organizations. The NFC Forum will provide access and support for name requests by these external organizations. This support can be enabled in a timely and responsive fashion as new objects and descriptions are produced. 
6. Security Considerations

There are no additional security considerations other than those normally associated with the use and resolution of URNs in general.

7. IANA Considerations

The requested NID ( $\mathrm{f} f \mathrm{C}$ ) has been entered into the IANA registry for URN NIDs.

8. Informative References

[RFC2141] Moats, R., "URN Syntax", RFC 2141, May 1997.

[RFC3406] Daigle, L., van Gulik, D., Iannella, R., and P. Faltstrom, "Uniform Resource Names (URN) Namespace Definition Mechanisms", BCP 66, RFC 3406, October 2002.

Acknowledgments

The author wishes to express thanks to Dwight Smith (Motorola) for his guidance on the URN registration process and for providing, by example, a registration that was worthy of emulation.

Author's Address

Miller Abel

Technical Committee Delegate, NFC Forum

Microsoft Corporation

One Microsoft Way

Redmond, WA 98052

EMail: TPMenfC-forum.org 
Full Copyright statement

Copyright (C) The IETF Trust (2006).

This document is subject to the rights, licenses and restrictions contained in BCP 78, and except as set forth therein, the authors retain all their rights.

This document and the information contained herein are provided on an "AS IS" basis and THE CONTRIBUTOR, THE ORGANIZATION HE/SHE REPRESENTS OR IS SPONSORED BY (IF ANY), THE INTERNET SOCIETY, THE IETF TRUST, AND THE INTERNET ENGINEERING TASK FORCE DISCLAIM ALL WARRANTIES, EXPRESS OR IMPLIED, INCLUDING BUT NOT LIMITED TO ANY WARRANTY THAT THE USE OF THE INFORMATION HEREIN WILL NOT INFRINGE ANY RIGHTS OR ANY IMPLIED WARRANTIES OF MERCHANTABILITY OR FITNESS FOR A PARTICULAR PURPOSE.

Intellectual Property

The IETF takes no position regarding the validity or scope of any Intellectual Property Rights or other rights that might be claimed to pertain to the implementation or use of the technology described in this document or the extent to which any license under such rights might or might not be available; nor does it represent that it has made any independent effort to identify any such rights. Information on the procedures with respect to rights in RFC documents can be found in BCP 78 and BCP 79 .

Copies of IPR disclosures made to the IETF Secretariat and any assurances of licenses to be made available, or the result of an attempt made to obtain a general license or permission for the use of such proprietary rights by implementers or users of this specification can be obtained from the IETF on-line IPR repository at http://www.ietf.org/ipr.

The IETF invites any interested party to bring to its attention any copyrights, patents or patent applications, or other proprietary rights that may cover technology that may be required to implement this standard. Please address the information to the IETF at ietf-ipreietf.org.

Acknowledgement

Funding for the RFC Editor function is currently provided by the Internet Society. 